\title{
Viabilidade econômica do plantio de pomar de Araucária para produção de pinhão
}

O objetivo desta pesquisa foi simular a viabilidade econômica no estabelecimento de pomar de sementes com plantas enxertadas de Araucária angustifolia para produção de pinhão na Região Metropolitana de Curitiba, estado do Paraná. Foram analisados três cenários: com aquisição de terra; com arrendamento de terra e sem aquisição ou arrendamento de terra. Os indicadores utilizados compreenderam a Taxa Interna de Retorno (TIR), o Valor Presente Líquido (VPL) e o Valor Esperado da Terra (VET). Utilizaram-se parâmetros de custo caixa e preço alvo de venda. Os resultados apontam que $75 \%$ dos VPL das simulações do cenário de compra de terra foram positivos, sendo o VPL médio de R\$17.499/ha, TIR 8,6\%a.a. e preço alvo de R\$3,16/kg. Para arrendamento $71 \%$ dos VPL das simulações foram positivos, o VPL foi de R\$14.996/ha, a TIR foi de $8,9 \%$ a.a. e o preço alvo médio foi de $R \$ 3,26 / \mathrm{kg}$. Sem compra de terra, não houve VPL negativo e sua média foi de $\mathrm{R} \$ 51.025 / \mathrm{ha}$, com TIR de 15,8\%a.a. e preço alvo de R\$1,79/kg. O VET médio foi de R\$51.189/ha produtivo. Encontrou-se grande variação nos indicadores em função do preço da terra, do nível de produção e preço do pinhão. 0 cenário mais arriscado foi o arrendamento, dado o maior custo fixo.

Palavras-chave: Ambiência; Conforto térmico; Construções Rurais; Perfil bioclimático.

\section{Economic feasibility of planting Araucaria orchard for jatropha production}

\begin{abstract}
The Parana pine (Araucaria angustifolia) is arguably the most important features of mixed ombrophilous forests in Southern Brazil. The species has great economic, ecological and cultural value in the region. Still, due to past overexploitation, only $2 \%$ of the original forest cover is left and the species is critically endangered. Hence, policies to recover forest cover and promote planting efforts are urgent. Here we investigate the economic feasibility of seed orchards established via grafted plants of Araucaria angustifolia for pine nut production in the metropolitan area of Curitiba - Brazil as means to incentivize the conservation of the species. We considered three options of establishment costs: 1) Land acquisition, 2) Land renting and 3) No land costs. To evaluate the economic feasibility of the orchards, we computed the internal rate of return (IRR), Net Present Value (NPV) and the Land Expectation Value (LEV) of the investment. The results show that the NPV was positive in $75 \%$ of the scenarios with land acquisition (17499 BRL/ha on average), with an $8.6 \%$ IRR and target price of $3.16 \mathrm{BRL} / \mathrm{kg}$. For the land renting case, the NPV was positive in $71 \%$ of the scenarios, with a NPV of $14996 \mathrm{BRL} / \mathrm{ha}$, IRR of $8.9 \%$ and target price of 3.26 $\mathrm{BRL} / \mathrm{kg}$. When no land costs were incurred, all NPV scenarios were positive (51025 BRL/ha, on average), the IRR reached $15.8 \%$ and the target price was 1.79 $\mathrm{BRL} / \mathrm{kg}$. Furthermore, the land renting option showed the highest NPV variability, due to the higher fixed costs incurred. We conclude that the promotion of Para pine orchards provide a suitable option to promote the expansion of forest area and generate extra income in rural areas.
\end{abstract}

Keywords: Extinction, Endangered species, Grafting

Topic: Uso de Recursos Naturais

Reviewed anonymously in the process of blind peer.
Received: 23/04/2021

Approved: 24/05/2021
Alan Lessa Derci Augustynczik (iD

Universidade Federal do Paraná, Brasil

http://lattes.cnpq.br/1432810740255965

http://orcid.org/0000-0002-7650-2782

alanlda@hotmail.com

\section{Gilson Luis Kolenez}

Universidade Federal do Paraná, Brasil

http://lattes.cnpq.br/6832730877875670

kolenez@gmail.com

\section{Ivar Wendling}

Embrapa Florestas, Brasil

http://lattes.cnpq.br/0359473031967049

ivar.wendling@embrapa.br

\section{Romano Timofeiczyk Júnior \\ Universidade Federal do Paraná, Brasil \\ http://lattes.cnpq.br/1246071381544725 \\ romano.timo@gmail.com}

Andrey Lessa Derci Augustynczi

Universidade Federal do Paraná, Brasil

http://lattes.cnpq.br/2038869207655454

andreylessa@gmail.com

\section{Dagma Kratz}

Universidade Federal do Paraná, Brasil

http://lattes.cnpq.br/1374045055025799

kratzdagma@gmail.com

\section{Referencing this:}

AUGUSTYNCZIK, A. L. D.; KOLENEZ, G. L.; WENDLING, I.; TIMOFEICZYK JÚNIOR, R.; AUGUSTYNCZIK, A. L. D.; KRATZ, D.. Viabilidade econômica do plantio de pomar de Araucária para produção de pinhão. Revista Ibero Americana de Ciências Ambientais, v.12, n.5, p.597-605, 2021. DOI: http://doi.org/10.6008/CBPC2179-6858.2021.005.0047 


\section{INTRODUÇÃO}

A Araucaria angustifolia, árvore símbolo do Estado do Paraná, é uma espécie cuja ocorrência se dá na Floresta Ombrófila Mista, que é uma das fitofisionomias do Bioma Mata Atlântica, considerado Patrimônio Nacional pelo art. 225 da Constituição Federal do Brasil de 1988. É uma espécie de grande importância social, econômica, cultural e ambiental, especialmente para a Região Sul do Brasil. Originalmente cobria grandes áreas contínuas na Região Sul do Brasil, estendendo-se para São Paulo, Minas Gerais, Rio de Janeiro e Espírito Santo na forma de pequenas manchas isoladas, principalmente nas regiões mais frias e altas destes estados (CARVALHO, 2003). De acordo com Hueck (1972), a araucária cobria uma área equivalente a $182 \mathrm{mil} \mathrm{km}^{2}$ nos planaltos brasileiros até o início do século XX.

No final do século XIX houve início de uma exploração desordenada da espécie, dado o alto valor de sua madeira (STEFENON et al., 2003). Isso fez com que a espécie entrasse para a lista oficial de espécies da flora brasileira ameaçadas de extinção, Figurando na Portaria MMA n43, de 17 de dezembro de 2014. Estima-se que da área original de floresta de araucária, que antes cobria as serras meridionais brasileiras, restaram apenas $2 \%$, tornando-se o ecossistema mais devastado do país (ZANETTE et al., 2017).

Além do valor da madeira, o pinhão, semente da araucária, possui grande importância econômica para os estados de ocorrência natural da araucária, sendo o pinhão comercializado oriundo, principalmente, de povoamentos naturais de Araucária (DANNER et al., 2012). Importante destacar que, conforme Silveira et al. (2011), o aumento dos preços do pinhão conduz a uma exploração mais intensa dos pinhais nativos, o que pode gerar problemas para a conservação da araucária. No mercado atacadista paranaense os preços do pinhão vêm subindo ano após ano (CEASA/PR, 2020).

No intuito de viabilizar o plantio de pomares de araucária com vistas à produção de pinhão, diversos estudos têm sido realizados no sentido de gerar conhecimento para a produção de mudas que incentivem o plantio da espécie, destacando-se o estabelecimento de protocolos de propagação vegetativa (WENDLING et al., 2009; ZANETTE et al., 2011; WENDLING et al, 2016; GASPAR et al., 2017; CONSTANTINO et al., 2018; RICKLI-HORST et al., 2019).

Para se ter dimensão do potencial produtivo, de acordo com Zanette et al. (2017) uma única araucária, localizada no município de Caçador-SC, chegou a produzir $160 \mathrm{~kg}$ de pinhão no ano de 2016. Por outro lado, em estudo realizado por Figueiredo Filho et al. (2011), foi observado que em populações naturais de araucária a produção foi da ordem de $11,4 \mathrm{~kg}$ de pinhão por árvore. Tendo em vista o mercado potencial para a comercialização do pinhão e a possibilidade de novas tecnologias de produção, esse trabalho teve como objetivo geral simular a viabilidade econômica do plantio de plantas enxertadas de Araucaria angustifolia para produção de pinhão na Região Metropolitana de Curitiba (RMC) nas modalidades de aquisição de terra, arrendamento de terra e sem o preço da terra.

\section{METODOLOGIA}

Para a realização desta pesquisa, foram utilizadas informações de custos e investimentos, obtidas por meio de consultas a viveiros e casas de insumos. A produtividade foi obtida com base em simulador 
elaborado a partir de informações disponíveis na literatura e experiência do autor especialista na área. Os preços foram coletados no banco de dados da SEAB/PR e CEASA/PR.

Todas essas bases referem-se ao plantio de um pomar de araucária para a produção de pinhão com espaçamento 10 x 10m (WENDLING, et al., 2017), nos municípios da Região Metropolitana de Curitiba (RMC). A RMC é composta por 29 municípios de acordo com a coordenação da região metropolitana de Curitiba (COMEC, 2020). Esta região foi selecionada por ser representativa da região fitogeográfica da Floresta Ombrófila Mista (ITCG, 2009) e por estar próxima a um importante centro consumidor de pinhão e de escoamento da produção para outras regiões consumidoras.

O preço bruto da terra ( $\mathrm{R} \$ \mathrm{~h} / \mathrm{ha}$ ) foi obtido com base na pesquisa de Terras Agrícolas realizada pela Secretaria de Estado da Agricultura e Abastecimento do Paraná (SEAB/PR, 2020), tendo como base terras para atividade de pecuária e reflorestamento - Classes A-IV e B-VI (FIGURA 1). Para as simulações foi utilizado o intervalo de preço referente a um desvio padrão para ou para menos, sendo o limite inferior $\mathrm{R} \$ 17.404 /$ ha e o limite superior $\mathrm{R} \$ 27.550 / \mathrm{ha}$.

Variação do preço da terra (R\$̦/ha) na RMC

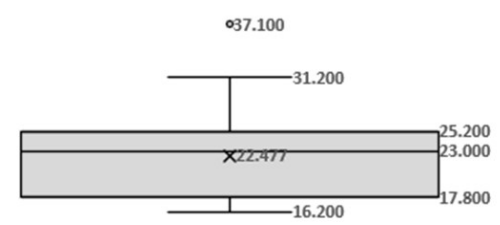

Figura 1: Variação do preço da terra (R\$̦/ha) na Região Metropolitana de Curitiba

Os preços do pinhão foram obtidos por meio do valor bruto da produção (VBP), calculado pela Secretaria de Estado da Agricultura e Abastecimento do Paraná (SEAB/PR, 2020) e pelos dados de comercialização das Centrais de Abastecimento do Paraná (CEASA/PR, 2020). O preço recebido pelo produtor no ano de 2020 foi calculado com base no mark up médio, obtido pela relação histórica (2012 a 2019) entre o Valor Bruto da Produção (VBP) e o valor praticado no CEASA/PR (Figura 2). O preço obtido foi de $R \$ 3,86 / \mathrm{kg}$ e, para fins de análise de sensibilidade, foi avaliado o intervalo de preço entre $R \$ 2,50 / \mathrm{kg}$ e $\mathrm{R} \$ 5,20 / \mathrm{kg}$.

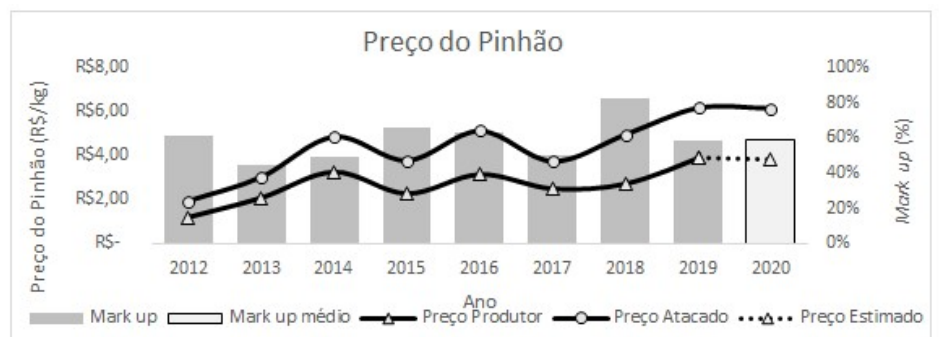

Figura 2: Mark up anual e médio e preço histórico anual e estimado do pinhão no produtor e no atacado.

A taxa mínima de atratividade (TMA) foi definida com base na taxa real oferecida pela letra do tesouro IPCA ${ }^{+} 2055$, que é de 3,99\%a.a (TESOURO DIRETO, 2020) mais um prêmio de risco com base na taxa EMBI+Risco Brasil, que entre janeiro e junho de 2020 foi de 3,32\% (IPEADATA, 2020). Dessa forma, a taxa mínima de atratividade, correspondente a taxa de juros real, foi de 7,44\%.

A curva de produção média por hectare foi obtida por meio de um simulador elaborado pelos 
autores. Visando analisar a sensibilidade da produção no desempenho da viabilidade econômica, foi estipulado um intervalo de análise para a realização do estudo (Tabela 1).

Tabela 1: Limites da curva de produção de pinhão (kg/ha).

\begin{tabular}{lcc}
\hline & Idade & \multicolumn{1}{c}{ Produção (kg/ha) } \\
\cline { 2 - 3 } & Limite Superior & Limite Inferior \\
\hline 0 a 7 anos & 0 & 0 \\
8 a 14 anos & 1.660 & 1.328 \\
15 a 19 anos & 2.490 & 1.992 \\
20 a 24 anos & 3.320 & 2.656 \\
25 a 30 anos & 5.810 & 4.648 \\
$>31$ anos & 6.640 & 5.312 \\
\hline
\end{tabular}

Para o cálculo dos impostos a serem pagos pelo investidor foi estabelecido o enquadramento tributário em regime Simples Nacional, CNAE (Código Nacional de Atividade Econômica) CNAE 0139-3/99, amparado pela Lei 10.406 de 2002 Código Civil (2020), e pelo Art. 165 da instrução normativa da Receita Federal do Brasil 971/2009 (RFB, 2020).

Para determinação da alíquota de imposto foi arbitrado um faturamento de até $\mathrm{R} \$ 180.000 / a n o$. Conforme determinação da Receita Federal Brasileira (RFB, 2020), a base de cálculo do imposto foi obtida através do cálculo da alíquota efetiva, multiplicando-se a receita bruta acumulada dos 12 meses anteriores pela alíquota indicada no anexo correspondente. Também foi aplicada a alíquota de 2,05\% sobre o faturamento bruto, referente ao FUNRURAL - Fundo de Assistência ao Trabalhador Rural.

Para o Imposto sobre Circulação de Mercadorias e Serviços (ICMS), o RICMS-PR, através do Decreto N.. 7.871, de 29.9.2017 abrange o produto deste estudo pelo diferimento. Art. 31, item 60 Paraná, (2020), portanto, não considerado nos cálculos.

Visando verificar a variação dos indicadores de viabilidade do investimento no pomar de araucária enxertada, foram analisados os seguintes cenários: a) Sem o preço de aquisição da terra: Realizadas simulações variando preço e produção de pinhão; b) Com o preço de aquisição da terra: Realizadas simulações variando preço do pinhão, produção de pinhão e preço da terra; c) Com arrendamento da terra: Realizadas simulações variando preço do pinhão, produção de pinhão e valor do arrendamento. Para identificar o retorno dos cenários propostos e de suas variações foram elaborados 1650 fluxos de caixa, que representam as projeções de entradas e saídas dos recursos ao longo do tempo (Figura 3).

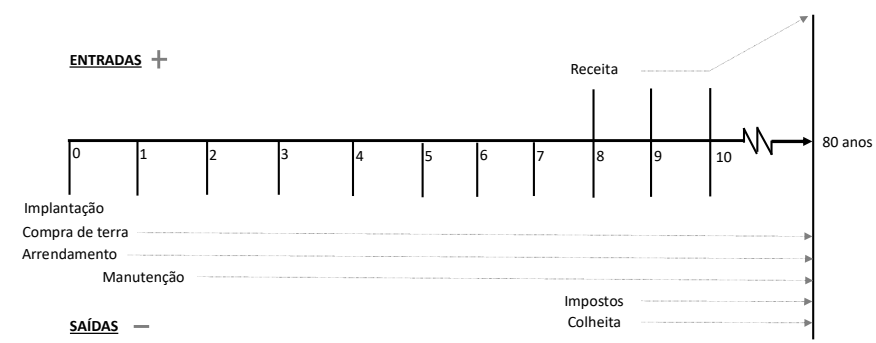

Figura 3: Representação esquemática dos fluxos de caixa dos cenários estudados.

Após a elaboração dos fluxos de caixa foram utilizadas ferramentas de engenharia econômica para avaliar a rentabilidade dos cenários propostos e suas respectivas variações. Os valores foram estimados com base no hectare produtivo de terra, sendo usado como base de conversão dos valores o grau de 
utilização da terra (GUT) (BERGER, 2011). A análise de viabilidade dos cenários propostos foi realizada com base nos métodos da Taxa Interna de Retorno (TIR), do Valor Presente Líquido (VPL) e do Valor Esperado da Terra (VET) (SILVA et al., 2005; CASAROTTO FILHO et al., 2008).

Para cada um dos cenários foi avaliado o custo caixa - total de saídas de caixa dividido pela produção durante a vida do projeto, o preço alvo - valor presente líquido das saídas de caixa ligadas à produção dividida pelo valor presente líquido dos volumes produzidos, acrescido dos impostos e taxas e, por fim, foi avaliada a probabilidade de viabilidade de cada um dos cenários.

\section{RESULTADOS E DISCUSSÃO}

\section{Mercado}

Por meio dos dados do Valor Bruto da Produção (VBP) gerados pela SEAB/PR (2020), disponíveis até o ano de 2018, é possível verificar que, considerando a produção entre 2014 e 2018, a produção anual média do Paraná está na casa de 3.573t. Projetando essa produção a um preço estimado de $R \$ 3,85 / \mathrm{kg}$ para o ano de 2020, o valor bruto da produção pode chegar a $R \$ 13,8$ milhões (Figura 4). De acordo com as análises realizadas, os municípios da RMC, área de abrangência do estudo, participam com apenas 10,5\% do VBP do pinhão do Estado do Paraná.

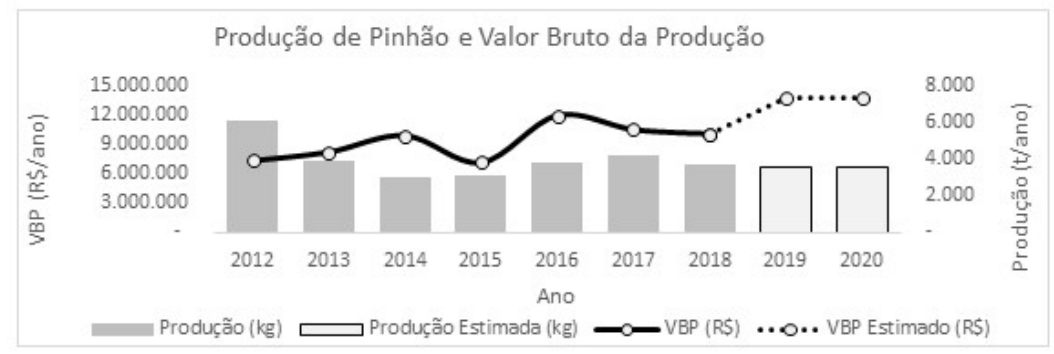

Figura 4: Valor bruto da produção (VBP) e produção de pinhão no Estado do Paraná, no período de 2012 a 2020.

Conforme pode ser observado na tabela 2, entre 2012 e 2019, cerca de 21,4\% do pinhão comercializado nas centrais de abastecimento do Paraná eram de procedência do Estado de Santa Catarina, outros $11,6 \%$ de Minas Gerais e 11,4\% de São Paulo (CEASA/PR, 2020).

Tabela 2: Procedência do pinhão em toneladas consumido no mercado atacadista (CEASA/PR) paranaense entre os anos de 2012 e 2019.

\begin{tabular}{|c|c|c|c|c|c|c|c|c|c|c|}
\hline Procedência & 2012 & 2013 & 2014 & 2015 & 2016 & 2017 & 2018 & 2019 & Média & $\%$ \\
\hline MG & 1,6 & 21,2 & 49,0 & 148,5 & 63,7 & 38,0 & 111,3 & 331,0 & 95,5 & $11,6 \%$ \\
\hline$P R$ & 570,5 & 420,1 & 185,4 & 347,1 & 520,7 & 691,5 & 194,2 & 703,9 & 454,2 & $55,0 \%$ \\
\hline RS & - & 0,6 & 1,7 & 10,2 & - & 3,1 & 4,4 & 0,7 & 2,6 & $0,3 \%$ \\
\hline SC & 69,6 & 86,4 & 388,1 & 118,2 & 68,0 & 100,5 & 395,0 & 187,9 & 176,7 & $21,4 \%$ \\
\hline SP & 103,0 & 139,9 & 169,8 & 85,5 & 91,6 & 22,7 & 46,7 & 95,5 & 94,3 & $11,4 \%$ \\
\hline Outros & 1,8 & - & 14,0 & - & - & - & 1,2 & 0,2 & 2,3 & $0,3 \%$ \\
\hline Total & 746,4 & 668,1 & 808,0 & 710,7 & 744,0 & 855,7 & 752,7 & $1.319,2$ & 825,6 & $100,0 \%$ \\
\hline
\end{tabular}

Cabe destacar também que o pinhão tem tido cada vez mais aplicações na culinária, tendo em vista o número crescente de receitas e produtos obtidos a partir do mesmo. Por ser considerado um alimento versátil, pode ser incorporado inteiro ou em pedaços aos pratos mas, também triturado, sendo 
efetivamente misturado a diversas receitas (GODOY et al., 2013).

\section{Custos e Preço Alvo}

O Grau de Utilização da Terra (GUT) usado para o cálculo do hectare produtivo foi de $66,8 \%$. Isso implica que para cada 1 ha produtivo, é necessário 1,5ha bruto. O custo de implantação do pomar de araucária foi de $\mathrm{R} \$ 8.134,00 / \mathrm{ha}$, sendo que $81 \%$ deste valor foi referente ao custo com a aquisição de mudas. Por se tratar de uma tecnologia nova, as mudas ainda apresentam preços elevados no mercado, o que eleva os custos de implantação. O custo de manutenção do empreendimento nos anos 1 e 2 do projeto foram de aproximadamente $R \$ 815,00 /$ ha.ano e a partir do ano 3 foi de $R \$ 765,00 /$ ha.ano. Salientase que cerca de $68 \%$ deste custo é referente a taxas bancárias, despesas de contabilidade e imposto territorial rural da propriedade. Os $32 \%$ restantes referem-se aos custos operacionais do empreendimento, tais como roçadas, manutenção de aceiros e fertilização, etc.

O custo caixa do pinhão $(\mathrm{R} \$ \mathbf{k g})$ variou de acordo com o nível de produção e preço da terra (FIGURA 5). O maior custo caixa do pinhão foram observados nas simulações do cenário de arrendamento, no qual o valor variou de $R \$ 1,51 / \mathrm{kg}$ até $R \$ 2,09 / \mathrm{kg}$. As simulações do cenário de compra de terra revelaram que o custo caixa do pinhão foi no mínimo de $R \$ 1,19 / \mathrm{kg}$ e no máximo de $R \$ 1,45 / \mathrm{kg}$.

Por fim, o custo caixa do cenário sem a compra de terra foi de $\mathrm{R} \$ 1,12 / \mathrm{kg}$ a $\mathrm{R} \$ 1,33 / \mathrm{kg}$. Por meio dessas análises foi possível verificar que o custo caixa do cenário de arrendamento é mais sensível ao nível de produção comparativamente aos demais cenários em função do seu maior custo fixo.

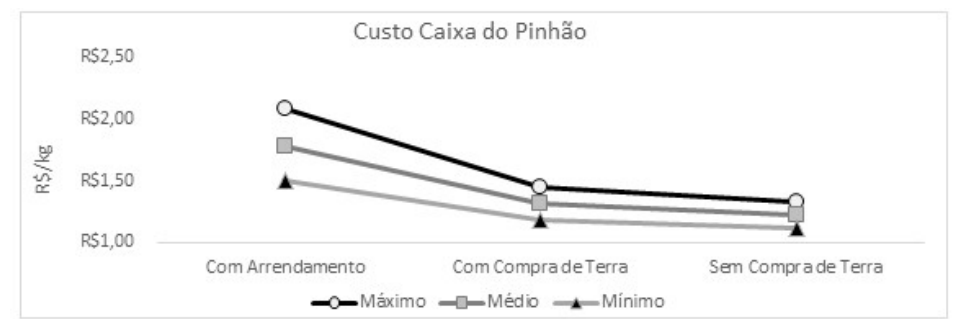

Figura 5: Custo caixa $(\mathrm{R} \$ / \mathrm{kg})$ máximo, médio e mínimo do pinhão para os cenários de arrendamento, compra de terra e sem compra de terra.

O preço alvo do pinhão $(\mathrm{R} \$ \mathrm{~kg})$ para que o investidor seja remunerado de acordo com a taxa mínima de atratividade e, considerando as premissas adotadas nesta pesquisa, variou de acordo com o nível de produção e preço da terra (Figura 6). Verificou-se que para o cenário de arredamento, o preço alvo foi maior do que nos demais cenários, variando de $R \$ 2,64 / \mathrm{kg}$ até $R \$ 3,97 / \mathrm{kg}$. O Preço alvo no caso da compra de terra foi de $\mathrm{R} \$ 2,58 / \mathrm{kg}$ a $3,84 / \mathrm{kg}$ e sem considerar a compra da terra o preço alvo foi de $\mathrm{R} \$ 1,63 / \mathrm{kg}$ até $\mathrm{R} \$ 1,96 / \mathrm{kg}$. A terra exerce grande pressão sobre o preço alvo de venda.

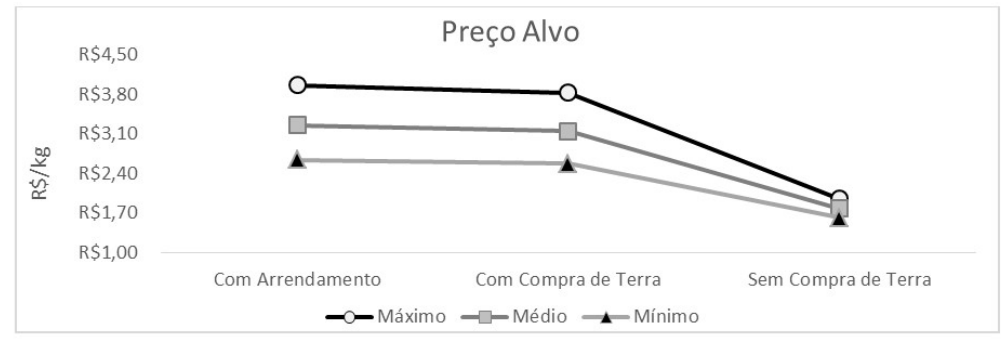

Figura 6: Variação do preço alvo (R\$/kg) máximo, médio e mínimo do pinhão para os cenários de arrendamento, 
compra de terra e sem compra de terra.

Essa análise demonstrou que a realização de uma boa gestão de custos e gestão da produção é fundamental para a viabilidade do projeto. Além disso, sugere-se especial atenção sobre o planejamento tributário, pois o peso do imposto sobre o custo da produção pode aumentar conforme o aumento do faturamento do empreendimento e isso pode alterar de forma significativa a viabilidade do projeto.

Portanto é recomendável avaliar outras opções de regime tributário, tais como as propostas por Tamarindo et al. (2020), que afirmam que o produtor rural pode estar enquadrado como produtor pessoa física e como pessoa jurídica nos regimes tributários: Simples Nacional, Lucro Presumido, Lucro Real ou Lucro Arbitrado.

\section{Análise de viabilidade e sensibilidade}

A partir das 1.650 simulações realizadas observou-se que para o cenário de compra de terra 75\% dos cenários avaliados apresentaram VPL positivo, sendo que na média o VPL foi de $\mathrm{R} \$ 17.499$. Para o cenário de arrendamento $71 \%$ dos cenários apresentaram VPL positivo, sendo que na média o VPL foi de $\mathrm{R} \$ 14.996$. Para o cenário sem compra de terra não houve VPL negativo a sua média foi de $\mathrm{R} \$ 51.025$ (Figura 7). Os cenários de compra de terra e arrendamento mostraram-se sensíveis ao nível de preço, sendo que a um preço de $\mathrm{R} \$ 2,80 / \mathrm{kg}$, observou-se uma probabilidade de geração de VPL negativo superior a $90 \%$. Por isso é de fundamental importância que sejam tomados todos os cuidados para garantir os níveis de produção e buscar alternativas para melhorar as condições de preço do produto.

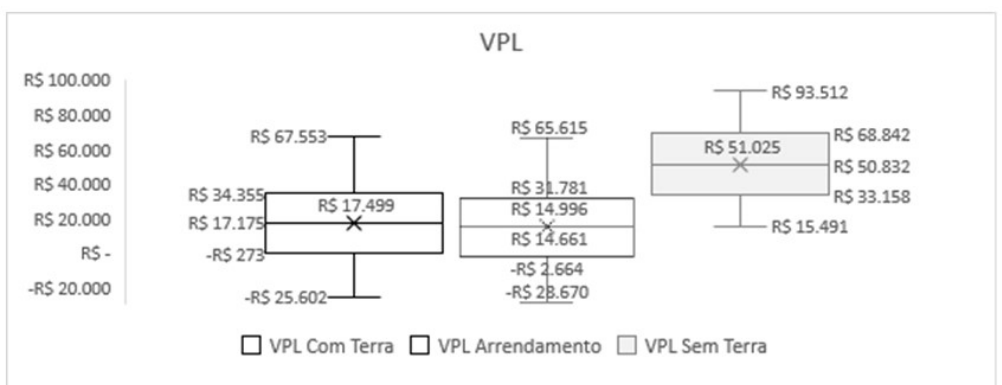

Figura 7: Sensibilidade do VPL em relação aos cenários avaliados de compra de terra, arrendamento e sem compra de terra.

Como era de esperar, a TIR para o cenário sem compra de terra apresentou o valor médio mais elevado, sendo 15,8\%a.a. O cenário que apresentou a menor TIR foi o de compra de terra, com uma média de 8,6\%а.a. O Cenário de arrendamento apresentou TIR média de 8,9\%а.a. A menor taxa interna de retorno observada foi de 3,5\%a.a. de juros real para o cenário de arrendamento (Figura 8).

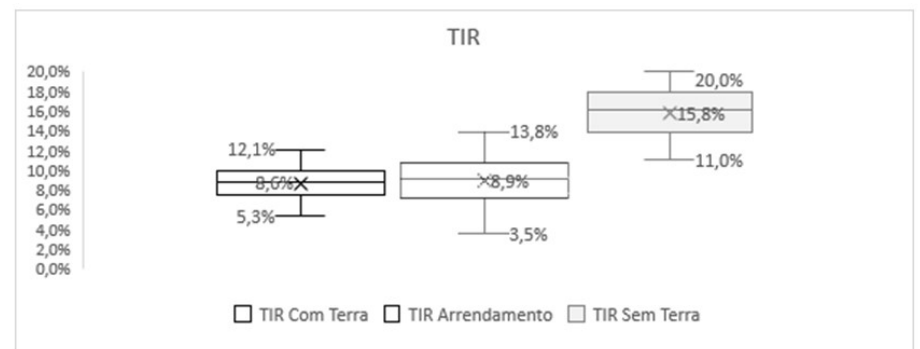

Figura 8: Sensibilidade da TIR em relação aos cenários avaliados de compra de terra, arrendamento e sem compra de terra. 
Para os parâmetros analisados, o valor médio esperado da terra foi de $\mathrm{R} \$ 51.189 /$ ha produtivo. Cerca de $76 \%$ das simulações realizadas apresentaram valores acima do valor médio do hectare produtivo da região analisada, que é de $\mathrm{R} \$ 33.633 /$ ha produtivo (Figura 9).

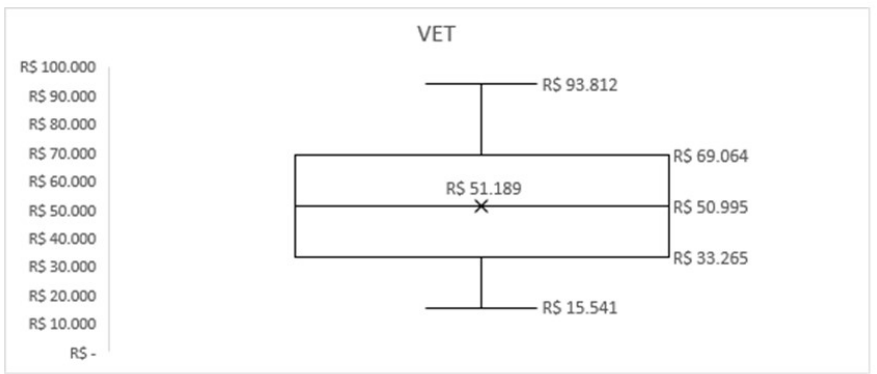

Figura 9: Variação do Valor esperado da terra (VET) nas simulações realizadas.

\section{CONCLUSÕES}

A partir das simulações realizadas, observou-se que há uma grande variação nos indicadores de rentabilidade em função do preço da terra, do nível de produção e do preço do pinhão. O cenário de arrendamento, apesar da vantagem de não imobilizar o capital na terra, se mostrou como o mais arriscado em função do maior custo fixo e, por consequência, da necessidade de maior preço de venda para garantir a rentabilidade do empreendimento. Produtores que pretendem converter a produção da propriedade para a produção de pinhão recomenda-se que avaliem os indicadores de rentabilidade da cultura atual comparativamente a viabilidade do pomar de araucária antes de seguir com a conversão.

Os tributos são parte importante da composição de custos e a alíquota se altera conforme o nível de faturamento no regime Simples Nacional. Por isso é recomendável avaliar os tributos caso a caso, pois podem alterar significativamente a viabilidade do projeto. Tanto o preço, quanto a produção do pinhão são componentes de grande impacto nos resultados financeiros do projeto, portanto são itens que exigem atenção e controle.

\section{REFERÊNCIAS}

BERGER, R.; SANTOS, A. J.; TIMOFEICZYK JÚNIOR, R.; BITTENCOURT, A. M.; SOUZA, V. S.; EISFELD, C. L.. O efeito do custo da terra na rentabilidade florestal: um estudo de caso para Santa Catarina. Floresta, v.41, n.3, p.599-610, 2011.

CARVALHO, P. E. R.. Espécies arbóreas brasileiras. Brasília: 2003.

CASAROTTO FILHO, N.; KOPITTKE, B. H.. Análise de investimentos: matemática financeira, engenharia econômica, tomada de decisão, estratégia empresarial. 10 ed. São Paulo: Atlas, 2008.

CEASA/PR. Centrais de Abastecimento do Paraná. Informações de Mercado. 2020.

BRASIL. Lei 10.406. Código Civil e normas correlatas. Brasília: DOU, 2020.

COMEC. Coordenação da Região Metropolitana de Curitiba. Municípios da Região Metropolitana da Curitiba. 2020.
CONSTANTINO, V.; ZANETTE, F.. Enxertia de propágulos trunciformes nos ramos de Araucaria angustifolia e multiplicação de matrizes. Ciência Florestal, v.28, n.2, p. 845-846, 2018.

BRASIL. Constituição da República Federativa do Brasi. Brasília: DOU, 1988.

DANNER, M. A.; ZANETTE, F.; RIBEIRO, J. Z.. O cultivo da araucária para produção de pinhões como ferramenta para a conservação. Pesquisa Florestal Brasileira, v.32, n.72, p.441451, 2012.

GOODOY, R. C. B.; NEGRE, M. F. O.; MENDES, L. M.; SIQUEIRA, G. L. A.; HELM, C. V.. O pinhão na culinária. Colombo: Embrapa Florestas, 2013.

FIGUEIREDO FILHO, A.; ORELLANA, E.; NASCIMENTO, F.; DIAS, A. N.; INOUE, M. T.. Produção de sementes de Araucaria angustifolia em plantio e em floresta natural no Centro-Sul do Estado do Paraná. Floresta, v.41, n.1, p.155- 
162, 2011.

GASPAR, R. G. B.; WENDLING, I.; STUEPP, C. A.; ANGELO, A. C.. Rootstock age and growth habit influence top grafting in Araucaria angustifolia. Cerne, v.23, n.4, p.465-471, 2017.

HUECK, K.. As florestas da América do Sul: ecologia, composição e importância econômica. São Paulo: Polígono, 1972.

IPEADATA. Instituto de Pesquisas Econômicas Aplicadas. Dados. 2020.

ITCG. Instituto de Terras, Cartografia e Geologia. Mapa Formações Fitogeográficas: Estado do Paraná. Curitiba, 2009.

MMA. Ministério do Meio Ambiente. Lista nacional das espécies da flora brasileira ameaçadas de extinção.

OLIVERA, F. C.; NOREÑA, C. P. Z.; PETTERMANN, A. C.; MARCZAK, L. D. F.. Influence of cooking in sorption isotherms of pinhão (Araucaria angustifolia seeds). Latin American Applied Research, v.42, n.1, p.11-18.

PARANÁ. LEI 11.580/1996. Dispõe sobre o ICMS no Estado do Paraná. Curitiba: DOE, 1996.

BRASIL. Receita Federal do Brasil. Simples Nacional, 2020.

RICKLI-HORST, H. C.; WENDLING, I.; KOEHLER, H. S.; ZUFFELLATO-RIBAS, K. C.. Araucaria angustifolia budding techniques in indoor and outdoor stablished rootstocks. Floresta e Ambiente, v.26, n.3, p.1-8, 2019.

SEAB. Secretaria Estadual de Agricultura e Abastecimento. Preços de Terras. 2020.

SICONTIBA. Sindicato dos Contabilistas de Curitiba e região. Tabela orientativa referencial de honorários.

SILVA, C. V.; REIS, M. S.. Produção de pinhão na região de Caçador-SC: aspectos da obtenção e sua importância para comunidades locais. Revista Ciência Florestal, v.19, n.4, p.363-374, 2009.

SILVA, M. L.; FONTES, A. A.. Discussão sobre os critérios de avaliação econômica: valor presente líquido (VPL), valor anual equivalente (VAE) e valor esperado da terra (VET). Revista Árvore, v.29, n.6, p.931-936, 2005

SILVEIRA, E. R.; JAMHOUR, J.; FERRONATO, M. L.; XAVIER, E.; CALGARO, E. V. S.. Situação das famílias na extração e comercialização do pinhão no Sudoeste do Paraná. Synergismus Scyentifica, v.6, n.1, 2011.

STEFENON, V. M.; NODARI, R. O.; REIS, M. S.. Padronização de protocolo AFLP e sua capacidade informativa para análise da diversidade genética em Araucaria angustifolia. Scientia Forestalis, n.64, p.163-171, 2003.

TAMARINDO, U. G. F.; PIGATTO. G.. Tributação no Agronegócio: uma análise geral dos principais tributos incidentes. 2 ed. Leme: JH Mizuno, 2020.

TESOURO DIRETO. Preços e taxas dos títulos IPCA, pré e pós-fixados. 2020.

WENDLING, I.; DUTRA, L. F.; HOFFMANN, H. A.; BETTIO, G.; HANSEL, F.. Indução de brotações epicórmicas ortotrópicas para a propagação vegetativa de árvores adultas de Araucaria angustifolia. Agronomía Costarricense, v.33, n.2, p.309-319, 2009.

WENDLING, I.; STUEPP, C. A.; ZUFFELLATO-RIBAS, K. C.. Araucaria angustifolia grafting: techniques, environments and origin of propagation material. Bosque, v.37, n.2, p.285293, 2016

WENDLING, I.; ZANETTE, F.; RICKLI, H. C.; CONSTANTINO, V. Produção de mudas de araucária por enxertia. In: WENDLING, I. ZANETTE, F.. Araucária: particularidades, propagação e manejo de plantios. Brasilia: Embrapa, 2017.

ZANETTE, F.; OLIVEIRA, L. S.; BIASI, L. A.. Enxertia de Araucaria angustifolia (Bertol.) kuntze nas quatro estações do ano. Revista Brasileira de Fruticultura, v.33, n.4, p.13641370, 2011

ZANETTE, F., DANNER, M. A.; CONSTANTINO, V.; WENDLING, I.. Particularidades, biologia reprodutiva e hábitos de crescimento em plantas de Araucaria angustifolia. In: WENDLING, I.; ZANETTE, F.. Araucária: particularidades, propagação e manejo de plantios. Brasilia: Embrapa, 2017.

A CBPC - Companhia Brasileira de Produção Científica (CNPJ: 11.221.422/0001-03) detém os direitos materiais desta publicação. Os direitos referem-se à publicação do trabalho em qualquer parte do mundo, incluindo os direitos às renovações, expansões e disseminações da contribuiç̃o, bem como outros direitos subsidiários. Todos os trabalhos publicados eletronicamente poderão posteriormente ser publicados em coletâneas impressas sob coordenação da Sustenere Publishing, da Companhia Brasileira de Produção Científica e seus parceiros autorizados. Os (as) autores (as) preservam os direitos autorais, mas não têm permissão para a publicação da contribuiçãa em outro meio, impresso ou digital, em português ou em tradução. 